\title{
Prevalencia de Maloclusión en niños de 6 A 12 años de la ciudad de Coronel Oviedo, Paraguay, Año 2016
}

\author{
J ulieta Méndez ${ }^{1,2}$, Rosario Rotela² , Ana Gonzalez \\ ${ }^{1}$ Universidad Nacional de Caaguazú, Instituto Regional de Investigación en Salud. Paraguay \\ ${ }^{2}$ Universidad Nacional de Caaguazú, Facultad de Odontología. Paraguay
}

Cómo referenciar este artículo/

How to reference this article:

Méndez J, Rotela R, González A. Prevalencia de Maloclusión en niños de 6 A 12 años de la ciudad de Coronel Oviedo, Paraguay, Año 2016. Mem. Inst. Investig. Cienc. Salud. 2020; 18(2): 86-92

\begin{abstract}
RE S U ME N
Las maloclusiones, según la Organización Mundial de la Salud (OMS), ocupan el tercer lugar como problemas de salud oral. El objetivo de este trabajo de investigación fue determinar la frecuencia de maloclusión presente en niños de 6 a 12 años en la ciudad de Coronel Oviedo, Paraguay en el año 2016. Se realizó un estudio observacional descriptivo con componente analítico en niños con el primer molar superior e inferior permanente en al menos una hemiarcada, que presentaron el consentimiento informado firmado por los padres. Las variables estudiadas fueron la prevalencia de maloclusión, relación molar y canina, sobremordida vertical y horizontal, apiñamiento y mordida cruzada. Los datos se cargaron en una hoja de cálculo de Excel y se analizaron con el programa Stata 14 . Se encontró maloclusión en $66 \%$ de los niños, $85,7 \%$ presentó clase molar y canina Clase 1, 44,6\% sobremordida horizontal (overbite) normal, 35,7\% sobremordida vertical (overjet) normal, 55,4\% apiñamiento, $8,9 \%$ presentó mordida cruzada, $75 \%$ perfil recto, y $62,5 \%$ de desviación de la línea media. Con respecto a la edad $(p=3,2)$ y el sexo $(p=0,4)$ no se encontraron asociaciones estadísticamente significativas en relación a la maloclusión. La frecuencia de maloclusión en escolares de la ciudad de Coronel Oviedo fue alta.
\end{abstract}

Palabras clave: maloclusión, niños, prevalencia.

\section{Malocclusion Prevalence in 6 to 12 years old children in the city of Coronel Oviedo, Paraguay, 2016}

\begin{abstract}
A B S T R A C T
Malocclusions, according to the World Health Organization (WHO), rank third as oral health problems. The aim of this research work was to determine the frequency of malocclusion present in 6 to 12 years old children in the city of Coronel Oviedo, Paraguay in 2016.A descriptive observational study with analytic component was performed in the children that had the first permanent upper and lower molar in at least one hemiarch, who presented the informed consent signed by the parents. The outcomes were malocclusion, molar and canine relation, overjet and overbite, crowding and crossbite. The data was loaded into an Excel spreadsheet and analyzed with the Stata 14 program. Malocclusion was found in $66 \%$ of the children, $85.7 \%$ presented molar and canine Class I, 44.6\% normal overbite, 35.7\% normal overjet, $55.4 \%$ crowding, $8.9 \%$ presented crossbite, $75 \%$ straight profile, $62.5 \%$ deviation from the midline. In relation to age $(p=3.2)$ and sex $(p=0.4)$, no statistically
\end{abstract}

Financiación: ninguna. Conflicto de intereses: ninguno.

Fecha de recepción: junio 2020. Fecha de aceptación: julio 2020

* Autor correspondiente: Julieta Méndez. Universidad Nacional de Caaguazú. Instituto Regional de Investigación en Salud. Dirección: Tuyuti y Mariscal Estigarribia, Coronel Oviedo, Paraguay. Phone: +595 985300046.

Email: julieta_mendez92@hotmail.com

Este es un artículo publicado en acceso abierto bajo una Licencia Creative Commons 
significant associations were found in relation with malocclusion. The frequency of malocclusion in schoolchildren from Coronel Oviedo, Paraguay was high.

Keywords: malocclusion, children, prevalence.

\section{NTRODUCCI ÓN}

Para la Organización Mundial de la Salud en su escala de prioridad relacionada con problemas de salud bucal, la maloclusión ocupa el tercer lugar, superada por caries dental y periodontopatías. Varios estudios epidemiológicos sostienen que las maloclusiones dentales constituyen el segundo problema de salud pública oral en la población pediátrica ${ }^{(1,2)}$. La Academia Estadounidense de Ortodoncia recomienda que a los 7 años, un niño debe hacerse un examen de ortodoncia por dos razones: la oclusión posterior se establece cuando erupcionan los primeros molares. En ese momento, uno puede evaluar las relaciones anteroposterior y transversal de la oclusión, así como descubrir cualquier cambio funcional o mordida cruzada. Por otro lado, los incisivos han comenzado a estallar y se pueden detectar problemas como hacinamiento, hábitos, mordiscos profundos, mordiscos abiertos y algunas discrepancias en la mandíbula ${ }^{(3)}$.

La maloclusión es una alteración de la oclusión, es una condición del desarrollo, en la mayoría de los casos se debe a una distorsión moderada del desarrollo normal. Las maloclusiones se consideran factores de riesgo para gingivitis, periodontitis, caries dental, disfunción de la articulación temporomandibular, así como alteraciones estéticas y funcionales. Una maloclusión es sinónimo de una disfunción que puede tener efectos sobre el habla, la deglución, la fonación y la estética de la persona que la padece ${ }^{(4-7)}$.

La prevalencia de maloclusión y la distribución de los diferentes tipos varían según la raza y el origen étnico. La influencia genética ha sido considerada uno de los factores etiológicos. Probablemente el ejemplo más famoso es el de la familia de los Habsburgo, donde al menos 33 de sus miembros tenían este problema. Carlos I de España (y $\vee$ de Alemania) fue uno de los más afectados, que además de tener una prominente clase III presentó un bocado abierto que le impidió hablar correctamente (8).

Este trabajo es de gran importancia porque, según estudios realizados en otros países, la maloclusión está presente en un alto porcentaje de la población y de los cuales los afectados generalmente no conocen esta patología, por lo tanto no realizan ningún tratamiento. A largo plazo no solo afecta la vida social del individuo sino que también altera la funcionalidad del sistema estomatognático. Además, consideramos que la recopilación de datos es importante en nuestra población, ya que no encontramos estudios basados en este tema en nuestra región.

El objetivo de este trabajo de investigación fue determinar la frecuencia de maloclusión presente en niños de 6 a 12 años en la ciudad de Coronel Oviedo en 2016.

\section{SUJ ETOS Y MÉTODOS}

Se realizó un estudio observacional descriptivo con componente analítico sobre la frecuencia de maloclusión en niños de 6 a 12 años, de sexo masculino y femenino que asistieron del primer al sexto grado en escuelas urbanas de la ciudad de Coronel Oviedo en 2016.

Se calculó un tamaño de muestra basado en una prevalencia de maloclusión del $72,2 \%$ encontrada en un estudio realizado en Brasil en $2009^{(9)}$. Con un nivel de confianza del $95 \%$, un error de muestreo del $5 \%$ y un ancho total del intervalo de confianza de 0.25 , los datos se transfirieron a la tabla de Hulley para encontrar el tamaño de muestra para las proporciones. Se obtuvo un resultado de 52 niños que serían parte de la investigación.

Se incluyeron a los niños con la presencia del primer molar superior e inferior permanente en al menos una hemiarcada, que presentaron el consentimiento informado firmado por los padres. La muestra se estratificó por escuelas. Posteriormente, por asignación proporcional, la muestra se distribuyó en los seis 
grados. Se distribuyeron consentimientos informados en cada escuela y el estudio se realizó posteriormente con 66 niños que lo presentaron, de los cuales 10 fueron excluidos debido a la falta de claridad en las fotografías, dejando los datos de 56 niños para el análisis.

Para la recopilación de datos, se preparó un registro clínico que contenía los datos de los estudiantes inspeccionados, nombre y apellido, edad, sexo y escuela, además se tomaron fotos intraorales y extraorales con dos cámaras de canon profesionales, dos espejos intraorales para tomas fotográficas y varios juegos de abrebocas e inspección bucal respetando los estándares de bioseguridad

Para determinar que el sujeto no presentaba maloclusión, se tuvieron en cuenta los siguientes aspectos: tener clase I molar y canina, overbite y overjet normal o en construcción, así como no presentar apiñamiento o mordida cruzada, caracterizándose dentro de la maloclusión si tiene al menos una de estas características.

Los datos se cargaron en una hoja de cálculo de Excel y se analizaron con el programa Stata 14. Se utilizó estadística descriptiva para el análisis de las variables, además de la prueba T Student y Chi cuadrado para analizar la relación de la edad y el sexo con la maloclusión, respectivamente.

Se respetaron los principios éticos de autonomía, justicia y no maleficencia. Los niños se beneficiaron con el diagnóstico de su condición bucal y con el tratamiento disponible en la Clínica Dental de la Facultad. Un consentimiento informado fue llenado y firmado por sus padres.

\section{RESULTADOS}

Un total de 56 niños de 6 a 12 años que asistieron del $1^{\circ}$ al $6^{\circ}$ grado participaron en el estudio. De la población de estudio, el 54\% eran mujeres. El 21,4\% tuvieron $7 \mathrm{u}$ 8 años (Tabla 1).

Tabla 1: Características demográficas de la población del estudio de maloclusión en niños de 6 a 12 años de la ciudad de Coronel Oviedo en 2016. n: 56

\begin{tabular}{ll}
\hline Características demográficas & $\mathbf{n}(\%)$ \\
\hline Sexo & \\
Masculino & $26(46,0)$ \\
Femenino & $30(54,0)$ \\
Edad & \\
6 & $8(14,3)$ \\
7 & $12(21,4)$ \\
8 & $12(21,4)$ \\
9 & $9(16,1)$ \\
10 & $6(10,7)$ \\
11 & $6(10,7)$ \\
12 & $3(1,68)$ \\
\hline \hline
\end{tabular}

Se observó maloclusión en 37 niños que correspondían al $66,1 \%$ de la población de estudio. Se observó que 48 niños $(85,7 \%)$ presentaron clase molar I, 48 niños $(85,7 \%)$ I canina, 25 niños $(44,6 \%)$ una sobremordida horizontal (overbite) normal. Se observó sobremordida vertical (overjet) normal en solo 20 niños (35.7\%). De la población del estudio 27 niños $(48,2 \%)$ presentaron apiñamiento anterior inferior, 5 niños correspondientes al $8,9 \%$ presentaron mordida cruzada, 42 niños $(75 \%)$ presentaron un perfil recto y 35 niños $(62.5 \%)$ presentaban desviaciones de la línea media. (Tabla 2). 
Tabla 2: Características de oclusión de la población del estudio de maloclusión en niños de 6 a 12 años de la ciudad de Coronel Oviedo en 2016. n: 56

\begin{tabular}{lr}
\hline Características de oclusión & $\mathbf{n}(\%)$ \\
\hline Maloclusion & \\
Si & $37(66,1)$ \\
No & $19(33,9)$ \\
Clase Molar & \\
I & $48(85,7)$ \\
II & $7(12,5)$ \\
III & $1(1,8)$ \\
Iase Canina & \\
II & $48(85,7)$ \\
III & $6(10,7)$ \\
Overbite & $2(3,6)$ \\
Normal & \\
Aumentado & $25(44,6)$ \\
Disminuido & $11(19,6)$ \\
En construcción & $2(3,6)$ \\
Overjet & $18(32,1)$ \\
Normal & \\
Aumentado & $20(35,7)$ \\
Disminuido & $14(25,0)$ \\
En construcción & $4(7,1)$ \\
Apiñamiento & $18(32,1)$ \\
Superior e inferior & \\
Superior & $1(1,8)$ \\
inferior & $3(5,3)$ \\
No & $27(48,2)$ \\
Mordida cruzada & $25(44,6)$ \\
Si & \\
No & $5(8,9)$ \\
Perfil & $51(91,1)$ \\
Recto & \\
Convexo & $42(75,0)$ \\
Concavo & $13(23,2)$ \\
Desviación de la línea media \\
Yes & $1(1,8)$ \\
No & \\
\hline & $35(62,5)$ \\
\hline & $21(37,5)$ \\
\hline
\end{tabular}

De acuerdo con la edad y la maloclusión, no se encontraron asociaciones estadísticamente significativas ( $p=3.2$; T student). Según el sexo y la maloclusión, no se encontró asociación estadísticamente significativa ( $p=0.4$; Chi cuadrado).

\section{SCUSIÓN}

En este estudio, la prevalencia de maloclusión fue del 66,1\%. Estos datos son similares al $52 \%$ encontrado por Reddy. Sin embargo es una prevalencia alta comparado con un estudio realizado por Dhar en India donde se observó un $26.06 \%$ y un estudio realizado en Cáceres Brasil en 2006 donde se observó un 31.1\%, al igual que el $32.5 \%$ encontrado por Correa en Brasil en $2013^{(1,10)}$. Estas diferencias podrían deberse a la raza y las características específicas de la población.

Entre la relación de tipo molar, la Clase I es la más frecuente en todo el mundo. Sin embargo, sabemos que hay varios tipos de subdivisiones de Clase I con diversas 
características clínicas y etiologías ${ }^{(3,8)}$. En un estudio realizado por Reddy en 2013 en Nalgonda India en niños de 6 a 10 años de edad, se observó una prevalencia del 48,3\% de la Clase I, similar al estudio realizado por Medina en Caracas en 2012 en pacientes pediátricos de 2 a 12 años donde se observó una prevalencia del 47,4\% y, a su vez, relacionada con un estudio realizado por Mora en Veracruz en 2012 en niños de 8 a 10 años donde se observó una prevalencia del $50 \%^{(1,2,11,12)}$. Todos estos resultados difieren de este estudio donde se encontró una mayor prevalencia correspondiente al $85.7 \%$.

Con respecto a la Clase II, Medina observó una prevalencia de $34.2 \%$ similar a la encontrada por Botero $32.5 \%$, cifras más altas que la observada por Plazas, que fue de $25 \%$, pero menor que la encontrada por Mora correspondiente al $50 \%$. La revista latinoamericana de Ortodoncia y Odontología Pediátrica publicó un estudio realizado en Venezuela en el período comprendido entre 2000 y 2007 en niños de 2 a 16 años de edad, donde se observó una prevalencia de Clase II correspondiente al $40 \%$ en ese momento. Estos datos difieren del $9 \%$ observado por López, que está más cerca del $12.5 \%$ encontrado en este estudio, que es similar al $13.9 \%$ encontrado por Reddy. Estas diferencias podrían deberse a la variabilidad de razas o rasgos antropológicos entre los países en los que se realizó cada uno de estos estudios $(1,2,11,12)$.

Con respecto a la clase III, Medina observó un resultado de $18.4 \%$, más alto que lo que Botero encontró $12.6 \%$, ambos más altos que el $7.8 \%$ observado por Reddy, mientras que López y Mora no encontraron ningún caso de este tipo de maloclusión. En contraste, en este estudio encontramos solo un caso de Clase III ${ }^{(1,2,11,12)}$.

Medina observó una prevalencia de overjet normal de $39.3 \%$ similar a este estudio donde se observó $35.7 \%$. Botero observó una la relación normal en el $68.6 \%$ de los sujetos. López observó un overbite en un $26 \%$ similar a este estudio donde se observó un 19,6\%, mientras que Mora mostró una cifra más alta correspondiente al $50 \%$. Creemos que estas variaciones con respecto a la relación incisiva podrían deberse al rango de edad estudiado, ya que en 18 sujetos de nuestra investigación aún no se pudo determinar la relación incisiva porque se hallaban en construcción ${ }^{(2,11,12)}$.

Mora observó tres casos de mordida cruzada en su estudio, mientras que en este estudio se observaron 5 casos, correspondientes al 8,9\% de la población. A su vez, Souki, en un estudio llevado a cabo en Belo Horizonte en 2008, encontró una prevalencia del $30 \%$ de mordida cruzada posterior, una cifra mucho más alta en comparación con este estudio y el de Reddy, que observó $3.7 \%^{(1,12,13)}$.

Mora informa una prevalencia de apiñamiento dental del 46,5\%, una cifra ligeramente inferior a la encontrada en este estudio correspondiente al 55,3\%. Un estudio realizado por Jagan en India en 2013 menciona que hay una mayor frecuencia de hacinamiento en las mujeres debido al menor tamaño de las mandíbulas en comparación con los hombres ${ }^{(12,14,15)}$.

Mencionando los tipos de Perfiles encontrados por Plazas en su estudio, se encontró que $58.33 \%$ de los estudiantes eran convexos, $37.50 \%$ rectos y $4.17 \%$ cóncavos. Cifras bastante diferentes a las encontradas en este estudio que corresponden al $23.2 \%$ para el perfil convexo solamente, $75 \%$ para el perfil recto y $1.8 \%$ para el perfil cóncavo. Diferencias que podrían deberse a las diferentes características morfológicas de la población ${ }^{(11)}$.

Mora en su estudio observó que el $10 \%$ de la población presentaba una desviación de la línea media, una cifra extremadamente baja en comparación con este estudio en el que se observó que el $62.5 \%$ de nuestra población lo presentó. Esto podría deberse a la pérdida prematura de dientes, produciendo migración dental y, por lo tanto, afectando la coincidencia de la línea media ${ }^{(12)}$.

Respecto a las diferencias de género, Mora encontró una mayor prevalencia en niños similar al estudio realizado por Brizon en 2013 en Brasil en niños de 12 años, mientras que en el Latin American Journal of Orthodontics and Pediatric Dentistry se menciona que el género donde el número más frecuente de maloclusiones es femenino, con un $52,3 \%$ similar al estudio realizado por Cartes, donde también mostró una ligera tendencia hacia una mayor prevalencia y gravedad en las mujeres. En este estudio, así como en el estudio realizado por Reddy, no hubo diferencias 
estadísticamente significativas, coincidiendo con el estudio realizado por Ramachandra en 2014 en India en niños de 8 a 13 años. Esto podría deberse a diferencias en las muestras de los diferentes estudios ${ }^{(1,3,11,12,16,17)}$.

En un estudio realizado en India en 2012 en niños de 4 a 6 años, se encontraron diferencias estadísticamente significativas en términos de edad, mientras que en este estudio, las diferencias no se debieron al rango estudiado o al número de sujetos ${ }^{(18)}$.

En síntesis, la frecuencia de maloclusión en escolares de la ciudad de Coronel Oviedo, Paraguay fue alta. Se halló alta prevalencia de apiñamiento inferior. La maloclusión no estuvo relacionada al sexo o la edad.

A pesar de las limitaciones del estudio, como el tamaño de muestra pequeño, se realizó con un cálculo de tamaño de muestra. Una de las fortalezas del estudio fue que el diagnóstico fue realizado por un especialista en el área. Los resultados de este estudio pueden servir como guía para recomendar un diagnóstico prematuro a los padres para un tratamiento rápido y desviación de la línea media. La maloclusión no estuvo relacionada al sexo o la edad.

\section{AGRADECI MI ENTOS}

A la Dra. Ana Amarilla y la Dra. Ana Benegas por el apoyo recibido para la investigación.

\section{REFERENCI AS BIBLI OGRAFI CAS}

1. Reddy ER, Manjula M, Sreelakshmi N, Rani ST, Aduri R, Patil BD. Prevalence of Maloclussion among 6 to 10 year old Nalgonda school children : J I nt Oral Health 2013; 5(6):49-54. Disponible en: file:///C:/Users/J ulie/Desktop/jioh-05-06049.pdf.

2. Medina Díaz A. Asociación entre agenesia, maduración dental y maloclusión en pacientes pediátricos. Caracas, Venezuela: Universidad Central de Venezuela. Publicación en Internet; 2012. Disponible en:

http://saber.ucv.ve/jspui/bitstream/ 123456

789/3683/1/T026800004023-0-

AC_MEDINA_trabajo_final_aprobado000.pdf.

3. Ramachandhra R, Saravanan R, Karthikeyan M, Vishnuchandran C, Sudeepthi. Prevalence of malocclusion and need for early orthodontic treatment in children: Journal of Clinical and Diagnostic Research [serial online] 2014 May [cited: 2014 Jun 7]; 8:ZC60-ZC61. Disponible en: http://www.jcdr.net/back_issues.asp?issn $=0$ 973-

$709 x \& y e a r=2014 \&$ month $=$ May $\&$ volume $=8 \& \mathrm{i}$ ssue $=5 \&$ page $=$ ZC60-ZC61\&id $=4394$. doi: $10.7860 / / C D R / 2014 / 8604.4394$

4. Arias Lazarte G, Navarrete Seminario F. Maloclusión Clase II División 1: Revista Médica Basadrina, 2012; 6(2):35-6. Disponible en:

http://www.unjbg.edu.pe/ revista-

medica/pdf/revistamV6-2. pdf\#page $=37$. doi: https:// doi.org/10.33326/26176068.2012.2. 488

5. Herrera Morales MR, Rojas Vargas MA, Canseco Jiménez J. Frecuencia de respiración oral en niños con maloclusión,
Revista Odontológica Mexicana. 2009; 13 (2): 91-8. Disponible en: http://www. medigraphic. com/pdfs/odon/uo2009/uo092d.pdf

6. Salvador Borrás S., Vincent Rossel C., Guía para la reeducación de la deglución atípica y transtornos asociados. Valencia: NauLibres; 2011.

7. Deepak Chauhan, VinodSachdev,Tripti Chauhan, Kamal K. Gupta. A study of malocclusion and orthodontic treatment needs according to dental aesthetic index among school children of a hilly state of India. J IntSocPrev Community Dent. 2013 Jan-J un; 3(1): 32-7. Disponible en: http://www.ncbi.nlm.nih.gov/pmc/article s/PMC3894101/. doi: 10.4103/22310762.115706.

8. Di Santi de Modano J, Vázquez VB. Maloclusión Clase I. Definición, Clasificación, Caracteristicas clínicas y tratamiento: Revista Latinoamericana de Ortodoncia y Odontopediatría, 2003. Disponible en: http://ortodoncia. ws/ publicaciones/2003/pdf /art8. pdf.

9. Martins G, Lima KC. Prevalence of malocclusions in 10-12 year old schoolchildren in Ceará, Brazil: Oral Health Prev Dent. 2009; 7(3): 217-23.

10. Correa-Faria P, Ramos L, Martins R, Vieira G, Marques LS. Malocclusion in preschool children: prevalence and determinant factors: Eur Arch Paediatr Dent. 2014; 15:89-96. doi.org/10.1007/s40368013-0069-9

11. Plazas Román J, Martínez Bermúdez O, Castro Pacheco L, Solana García A, Villalba Manotas $L$. Prevalencia de maloclusiones en niños de una escuela en Cartagena de Indias 
Ciencia y Salud virtual.2011. 3 (1) 2-11 https: //doi.org/10.22519/21455333.38 12. Mora Morales C. Prevalencia de Maloclusiones en niños de 8 a 10 años que asisten a la escuela primaria Juan Escutia Poza Rica Veracruz. Veracruz [Tesis doctoral ]. Universidad Veracruzana Facultad de Odontología; 2012.

13. Souki B, Pimenta G, Souki M, Franco L, Becker $\mathrm{H}$, Pinto $\mathrm{J}$. Prevalence of malocclusion among mouth breathing children: do expectations meet reality. International Journal of Pediatric Otorhinolaryngology 73 (2009) 767-77. doi: 10.1016/j.ijporl.2009.02.006

14. Jagan K, Amrita G, Clement R, Anil T. Prevalence of malocclusion and its relationship with caries among school children aged 11 - 15 years in southern India: Korean J Orthod. Feb 2013; 43(1):35$41 . \quad$ Disponible en: http://synapse.koreamed.org/DOIx.php?id= 10.4041/kjod.2013.43.1.35

15. Isper Garbin Artênio José, Saliba Garbin Cléa Adas, Pantaleão dos Santos Maria Rita, Elaine Gonçalves Patrícia. Prevalencia de maloclusión en la dentición primaria en el municipio de Cáceres, Brasil. Rev Cubana Estomatol [Internet]. $2007 \quad$ Mar [citado 2020 Ago 25] ; 44( 1 ). Disponible en: http://scielo.sld.cu/scielo.php?script=sci_art text\&pid =S0034-

$75072007000100004 \&$ lng $=$ es.

16. Cartes Velasquez R, Araya E Valdéz C. Maloclusiones y su impacto psicosocial en estudiantes de un liceo intercultural: Int J Odontostomat, 2010; 4 (1): 65-70. Disponible en: http://www.scielo.cl/pdf/ijodontos/v4n1/art1 1.pdf.

17. Deepak P, Tarulatha R, Uma B, Shivaprakash. Study of occlusal characteristics of primary dentition and the prevalence of maloclusion in 4 to 6 years old children in India: Dental Research Journal, North America, 9, jan. 2013. Disponible en: <http://drj.mui.ac.ir/index.php/drj/article/vi ew/798/550>. doi: $10.4103 / 1735-$ $\underline{3327.104883}$

18. Brizon V, Cortellazzi K, Vazquez $F$, Ambrosano G, Pereira A, Gomes V, et al. Fatoresindividuais e contextuaisassociados a maoclusaoemcriancas brasileiras.: ev. Saúde Pública [serial on the Internet]., 2013 Dec [cited 2014 June 08] ; 47( Suppl 3 ): 118$128 . \quad$ Disponible en: http://www.scielo.br/scielo.php?script=sci_a rttext\&pid=S0034$89102013000900118 \&$ lng $=$ en. Doi: $10.1590 / S 0034-8910.2013047004426$ 